\title{
Study of Liver Function Test, Haematological Parameters and Crp Derangements in Covid-19 Patients
}

\author{
Gangaram Bhadarge 1 , Nandkishor Bankar² and Saurabh Hadke ${ }^{3}$ \\ ${ }^{1}$ Dept. of Biochemistry Jawaharlal Nehru Medical College, Datta Meghe \\ Institute of Medical Sciences Sawangi (Meghe) Wardha \\ ${ }^{2}$ Dept. of Microbiology Jawaharlal Nehru Medical College, Datta Meghe \\ Institute of Medical Sciences Sawangi (Meghe) Wardha \\ ${ }^{3}$ Dept. of General Medicine DattaMeghe Medical College, Nagpur, India \\ Corresponding author email: drbankarnj28@gmail.com
}

\section{ABSTRACT}

Covid-19 are $65 \mathrm{~nm}$ to $125 \mathrm{~nm}$ in diameter and contain single-stranded RNA as nuclei content. Covid-19's global scale transmission, frequent occurrence, large number of deaths The virus was primarily transmitted from person to person by physical interaction. Coughing, sneezing, and laughing cause droplets. Fever, chills, cough, sore throat, breathing difficulties, myalgia or fatigue, nausea, vomiting, and diarrhoea are common signs of COVID-19 infection. Study Of Liver Function Test, Haematological Parameters And Crp Derangements in Covid-19 patients 25 patients were ICU and 25 patients were ward. Patients went directly to the Observed Treatment in the Dept. of Medicine and Dept. of Respiratory, Datta Meghe Medical College and Shalinitai Meghe Hospital and Research Centre, However differences between the ICU and Ward liver function test, LDH ferritin and CRP patients were $(\mathrm{P}<0.0001)$ statistically significant Excepts total protein and albumin are not significant. $(\mathrm{P}=0.4403, \mathrm{P}=0.3465)$ The literature considers the deranged values of liver function enzymes in Covid-19 to be of less concern, while our study shows a connection between liver enzymes and the increased number of ICU admissions as well as prognostic markers, necessitating further research in this area to document the derangements of liver enzymes during hospitalization and their relationship to clinical outcomes.

KEY WORDS: COVID-19, SARS-COV-2,RNA, NSAIDS, DVT

\section{INTRODUCTION}

The coronavirus disease-2019 (COVID-19) epidemic first surfaced at the end of December in a Hunan seafood market in Wuhan, China, and was quickly proclaimed an international public health emergency by the World Health Organization (WHO, 2019). It's an infectious disease caused by the coronavirus-2 that causes extreme acute respiratory syndrome (SARS-CoV-2) SARS-CoV-2

Biosc Biotech Res Comm P-ISSN: 0974-6455 E-ISSN: 2321-4007

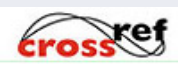

Identifiers and Pagination

Year: 2021 Vol: 14 No (6) Special Issue

Pages: $36-40$

This is an open access article under Creative

Commons License Attribn 4.0 Intl (CC-BY).

DOI: $h t t p: / / d x . d o i . o r g / 10.21786 / b b r c / 14.6 .8$ is phylogenetically related to SARS viruses, according to genomic studies, and bats may be the primary vector. While the virus's intermediate point of origin and spread to humans is unknown, it has been shown that it is capable of rapid human-to-human transmission. The virus was primarily transmitted from person to person by physical interaction. Coughing, sneezing, and laughing cause droplets. Fever, chills, cough, sore throat, breathing difficulties, myalgia or fatigue, nausea, vomiting, and diarrhoea are common signs of COVID-19 infection. Cardiovascular damage, respiratory failure, acute respiratory distress syndrome, and even death may occur in severe situations (Huang et al., 2020; TanjenaRumea et al., 2020).

Covid-19 is $65 \mathrm{~nm}$ to $125 \mathrm{~nm}$ in diameter and contains single-stranded RNA as nuclei content. Covid-19's global scale transmission, frequent occurrence, large number of

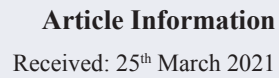

ccepted after revision: $2^{\text {th }}$ May 2021 
deaths, infection and mortality among care providers, and multiplicative impact in vulnerable or sensitive groups are all major causes of concern (Lokhandwala and Gautam, 2020).

SARS-CoV-2 infects endothelial cells in a variety of tissues, resulting in diffuse lymphocytic endotheliitis and vasoconstriction (Varga et al., 2020). Hypoperfusion occurs as a result of the inflammation, hypercoagulability, and edema that accompany it, resulting in organ ischemia. COVID-19, on the other hand, has little effect on patients with pre-existing immune-mediated inflammatory disorder who are being treated with anticytokine biologics and other immunomodulatory therapies. COVID-19 does not have a lot of bleeding. There are reports of deep vein thrombosis (DVT), venous thromboembolism (VT), pulmonary embolism (PE) and corpulmonale, systemic and pulmonary arterial thrombosis and embolism, ischemic stroke, and myocardial infarction (MI). Among the dead, DVT and PE are popular. Inflammation, platelet activation, hypercoagulability, endothelial dysfunction, blood vessel constriction, stasis, hypoxia, muscle immobilization, and disseminated intravascular coagulation are all factors that contribute to this (DIC) Hypercoagulability and impaired fibrinolysis are caused by fever and inflammation.

Ages of the cytokine interleukin-6 (IL-6) are linked to hypercoagulability and disease severity. Antiphospholipid antibodies that are elevated are linked to thrombosis. Procoagulant agents are produced in greater quantities by the liver. The time to prothrombin and the time to active partial thromboplastin are also moderately long. There is moderate thrombocytopenia. The level of C-reactive protein is high. Lymphocytopenia, elevated D-dimer, elevated fibrin degradation products (FDPs), and DIC are all linked to cytokine storm and excessive systemic inflammation. D-dimer and DIC amounts are prognostic. Patients with COVID-19 include subpleural and lateral regions with ground-glass opacity and consolidation on chest CT scans. The majority of the patients have distribution from both sides. Patchy infiltrates can be seen on chest radiographs, and they can be scattered asymmetrically (Jain, 2020).

Rhabdomyolysis, which can be caused by hyperventilation or drugs like remdesivir, can also induce acute kidney damage. In New York, about 90\% of mechanically ventilated patients developed AKI. AKI happens at the same time as respiratory failure (Hirsch et al., 2020).

Extra-pulmonary pathological manifestations of deranged liver function enzymes are prominently observed in residents suffering from extreme acute respiratory syndrome coronavirus disease 19 (SARS Covid-19), which is identified by roughly half of the patients. According to previous studies, 15 percent to 45 percent of patients experience symptoms of liver injury during infection. In patients in intensive care units (ICUs) and emergency states, biochemical markers in the liver are more likely to be abnormal, indicating the seriousness of infection.SARS-COVID 19 pathogenesis ensures the use of the angiotensin-converting enzyme 2 (ACE-2) receptor as a target for cell entry; this receptor plays a critical role in virus replication as it is scattered on endothelial cells of the bile duct and liver, rendering the liver a prominent organ to be infected. Moderate micro vascular steatosis and slight lobular and portal activation were seen in post-mortem results in deceased patients, indicating that dysfunction may be the result of COVID19 drug-potentiated hepatic damage.Other histological post-mortem findings point to liver enlargement, liver cell degeneration, lobular focal necrosis with neutrophil and lymphocyte aggregations in the portal region, and hepatic sinus obstruction with micro-thrombosis. Extreme pneumonia is a clinical symptom of COVID-19 infection in people who have deranged liver function enzymes.

Nonsteroidal anti-inflammatory drugs (NSAIDs), antibiotics, antiviral agents like ribavirin, lopinavir, ritonavir, herbal drugs, and interferon, all of which cause liver damage, increase the severity of infection. Uncontrollable immune manifestation and cytokines storm mediated systemic inflammation results in injury to the liver resulting in derangement of biochemical markers of liver, individuals suffering from COVID-19 were detected with high levels of TH17, CD8 T cells, interleukin-2, interleukin-6, interleukin-7, interleukin10 , tumor necrosis factor-alpha (TNF-alpha), granulocytecolony stimulating factor, interferon-inducible protein 10 , high levels of C-reactive protein in samples of their peripheral blood triggering dysfunction of liver enzymes (Fan et al., 2020).

Aim: Study Of Liver Function Test, Haematological Parameters And Crp Derangements In Covid -19 patients

\section{MATERIAL AND METHODS}

The study conducted Department of Biochemistry this study included 50Covid positive patients of age 40 -55 years who were admitted in ICU and Ward to Shalinitai Meghe hospital and Research centre, consequently, 25 patients were ICU and 25 patients were ward. Patients went directly to the Observed Treatment in the Dept. of Medicine and Dept. of Respiratory, DattaMeghe Medical College and ShalinitaiMeghe Hospital and Research Centre.

Sample Collection: $5 \mathrm{ml}$ of each patient's blood sample was taken and separated in two tubes EDTA and plain tube. The sample was used to estimate the levels of Liver function test, Ferritin, LDH, CRP and CBC.

Biochemical Analysis: EDTA samples were used for the CBC count was estimated on 3 parts coulter counter. The sample was used to estimate the levels of Liver function test, CRP, ferritin, LDH were estimated on AU480 Analyser.

\section{RESULTS}


Table 1. Comparison between ICU and Ward Covid-19 positive patients

\begin{tabular}{|l|c|c|c|}
\hline Parameters & ICU ( n-25) & Ward ( n-25) & P-valve \\
\hline Hb (gm/dl) & $11.20 \pm 3.1$ & $14.2 \pm 2.60$ & $\mathrm{P}=0.0005$ \\
\hline WBC (\%) & $14001 \pm 46$ & $9301 \pm 23$ & $\mathrm{P}<0.0001$ \\
\hline PLT(Laks/cumm) & $140.13 \pm 21.0$ & $212.10 \pm 34.7$ & $\mathrm{P}<0.0001$ \\
\hline Total Bilirubin (mg/dl) & $0.43 \pm 0.24$ & $1.54 \pm 1.41$ & $\mathrm{P}=0.0003$ \\
\hline Direct (mg/dl) & $0.20 \pm 0.15$ & $0.78 \pm 1.01$ & $\mathrm{P}=0.0066$ \\
\hline Indirect (mg/dl) & $0.23 \pm 0.9$ & $0.76 \pm 0.43$ & $\mathrm{P}=0.0107$ \\
\hline SGOT (IU/L) & $97.65 \pm 32.0$ & $56.31 \pm 23.0$ & $\mathrm{P}<0.0001$ \\
\hline SGPT (IU/L) & $110.1 \pm 26.0$ & $61.42 \pm 28.9$ & $\mathrm{P}<0.0001$ \\
\hline Total Protein (mg/dl) & $6.61 \pm 3.24$ & $6.18 \pm 1.34$ & $\mathrm{P}=0.4403$ \\
\hline Albumin (mg/dl) & $3.80 \pm 2.85$ & $3.24 \pm 1.27$ & $\mathrm{P}=0.3465$ \\
\hline Alkaline P (IU/L) & $166.4 \pm 65.10$ & $89.7 \pm 33.0$ & $\mathrm{P}<0.0001$ \\
\hline GGT (IU/L) & $93.7 \pm 35.0$ & $49.0 \pm 7.60$ & $\mathrm{P}<0.0001$ \\
\hline LDH (IU/L) & $487.41 \pm 34.0$ & $241.0 \pm 36.0$ & $\mathrm{P}<0.0001$ \\
\hline CRP (mg/L) & $57.0 \pm 9.2$ & $21.0 \pm 4.5$ & $\mathrm{P}<0.0001$ \\
\hline Ferritin (ng/dl) & $785.60 \pm 63.0$ & $483.2 \pm 48.3$ & $\mathrm{P}<0.0001$ \\
\hline
\end{tabular}

Comparison of $\mathrm{HB}, \mathrm{WBC}$ and Platelets in ICU patients was raised and ward patients were normal.

Comparison of liver function testsparameters in the study groups. Total and direct serum bilirubin levels were increased in ICU patient's normal in ward patients. The primary liver enzymes ALT and AST were raised in the both ICU and ward patients. Between the ICU and ward were ALKP was within normal in ward patients but raised in ICU patients. Serum Total Protein and Albumin levels were within normal limits in both ICU and ward the groups. The both groups differences were statistically not significant $(\mathrm{P}=0.4403, \mathrm{P}=0.3465)$.

Comparison between ICU and ward serum LDH level were more than ICU patients to ward patients. Comparison of serum Ferritin level is both ICU and ward patients are raised in both groups. Comparison CRP level in both ICU and Ward patients more than ward patients CRP level are high in ICU patients. However differences between the ICU and Ward liver function test, LDH ferritin and CRP patients were $(\mathrm{P}<0.0001)$ statistically significant Excepts total protein and albumin are not significant. $(\mathrm{P}=0.4403, \mathrm{P}=0.3465)$.

\section{DISCUSSION}

Multiple organs in different patients can be affected, with a temporal path unrelated to viral load. Complications are caused by inflammation, platelet activation, hypercoagulability, endothelial dysfunction, blood vessel constriction, stasis, hypoxia, and muscle immobilization. Lungs are often affected. There may be an Acute Coronary Syndrome, cardiac disease, or myocarditis present. Angiotensin-converting enzyme inhibitors and angiotensin II receptor antagonists can be continued by patients.AKI is normally caused by structural problems. There are cases of meningitis, encephalitis, encephalopathy, stroke, and delirium. The senses of smell and taste have been shown to be impaired. Viruses enter the body by the eyes, and may also be a cause of infection. Patients and caregivers alike suffer from psychological issues. There are signs of gastrointestinal distress. The most prominent skin symptom is a patchy erythematous rash. As a result, COVID-19 can damage almost every organ in the body (Jain, 2020).

The symptoms of AIL found in COVID-19 patients resemble Virchow's triad: altered blood flow, irregular platelet activity, and vascular endothelium, both of which can contribute to venous and arterial thrombosis (Hawale and Bhatt, 2021). In the sense of systemic microvascular thrombosis, mild thrombocytopenia has been reported in 5 to $20 \%$ of infected patients. Platelet levels in our hospitalized patients were below reasonable limits (Panigada et al., 2020). a study of thromboelastography results and other haemostasis parameters in covid-19 patients in the intensive care unit. In COVID 19 admitted patients, liver disease is normal, though it seems to be more common in COVID 19 serious cases. Severe liver injury that resulted in liver damage, liver failure, or death was previously unheard of. In 16 to $53 \%$ of patients, elevated ALT and AST levels were found. Chen et al. from Wuhan is the first to record abnormal liver enzymes in COVID 19 patients. In 43.4 \%of cases, he showed a rise in serum levels of ALT, AST, and LDH (Ghoda and Ghoda, 2020).

These conclusions are supported by the present study's findings. All three classes of admitted COVID-19 cases had elevated ALT and AST levels. The increase was about two times the upper limit of average. There were no statistically meaningful variations between the parties. Serum albumin levels were lower in the new research classes, with those admitted to the intensive care unit 
having the lowest levels. Since the serum total protein levels were normal, the drop in serum albumin may be attributed to the patients' poor nutritional status, which formed during their sickness or extended hospital stay (Gedam, Sachin Ratan et al, 2019).

In COVID-19, abnormal LFT is often temporary and occurs in conjunction with elevated enzymes from the heart and muscle, and it returns to normal without causing any liver-related morbidity or mortality (Husain et al., 2020).COVID-19 15Aminotransferase elevation may also be attributed to myositis, which is close to heavy influenza infection. 8 SARS-CoV-2 is thought to bind directly to cholangiocytes, demonstrating the Angiotensin Converting Enzyme 2 (ACE) receptor and causing liver damage, according to a recent report. 21 This helps to understand how SARSCoV-2 infection contributed to our patients' liver dysfunction (Chai et al., 2020).

Patients with irregular liver test results, particularly hepatocyte or mixed form (i.e., raised ALT/AST, or both ALT/AST and ALP/GGT) at admission or during hospitalization, had substantially higher chances of advancing to extreme COVID-19 than those with regular liver tests. Exacerbation of pneumonia to moderate pneumonia is a critical clinical outcome that indicates a higher mortality rate and necessitates ICU or mechanical ventilation. Age, ethnicity, and underlying diseases have all been identified as risk factors for extreme COVID19 in previous studies.This is one of the first studies to link pathological liver testing to serious illness. The SARS-CoV-2 virus is thought to be not only extremely transmissible, but also capable of causing extreme multiorgan dysfunction in humans, and our findings support this theory to some degree (NandkishorBankar et al., 2020; Prakashrao et al., 2020).

In our research, we found increased total leukocyte counts and elevated total leukocyte counts in COVID-19 patients with abnormal liver function parameters at the time of admission, which corresponded to the findings of a few studies (Bawiskar et al., 2020).

\section{CONCLUSION}

The literature considers the deranged values of liver function enzymes in Covid-19 to be of less concern, while our study shows a connection between liver enzymes and the increased number of ICU admissions as well as prognostic markers, necessitating further research in this area to document the derangements of liver enzymes during hospitalization and their relationship to clinical outcomes.

\section{REFERENCES}

Asghar, M.S., Akram, M., Rasheed, U., Hassan, M., Iqbal, Z., Fayaz, B., Hayat, H., Ather, A., Hussain, H. and Syyed, E., 2020. Derangements of Liver enzymes in Covid-19 positive patients of Pakistan: A retrospective comparative analysis with other populations.
Archives of Microbiology \& Immunology, 4(3), pp.110120.

Bankar, N., Ambad, R.S., Chandi, D.H. and Meshram, S., 2020. COVID-19 review: with special reference to laboratory diagnosis and testing strategy. Journal of critical reviews, 7(10), pp.187-192.

Bawiskar, N., Andhale, A., Hulkoti, V., Acharya, S. and Shukla, S., 2020. Haematological Manifestations of Covid-19 and Emerging Immunohaematological Therapeutic Strategies. Journal of Evolution of Medical and Dental Sciences, 9(46), pp.3489-3495.

Chai, X., Hu, L., Zhang, Y., Han, W., Lu, Z., Ke, A., Zhou, J., Shi, G., Fang, N., Fan, J. and Cai, J., 2020. Specific ACE2 expression in cholangiocytes may cause liver damage after 2019-nCoV infection. biorxiv.

Fan, Z., Chen, L., Li, J., Cheng, X., Yang, J., Tian, C., Zhang, Y., Huang, S., Liu, Z. and Cheng, J., 2020. Clinical features of COVID-19-related liver functional abnormality. Clinical Gastroenterology and Hepatology, 18(7), pp.1561-1566.

Gedam, S.R., Dhabarde, A., Patil, P.S., Sharma, A., Kumar, K. and Babar, V., 2019. Psychiatric Comorbidity, Severity of Dependence and Liver Enzymes Dysfunction among Alcohol Dependent Individuals: A Crosssectional Study from Central Rural India. Journal of Clinical \& Diagnostic Research, 13(4).

Ghoda, A. and Ghoda, M., 2020. Liver Injury in COVID19 Infection: A Systematic Review. Cureus, 12(7).

Hawale, D., Bhatt, N., 2021. Analysis of the Relationship Between Clinical Results and Laboratory Studies of Adult Patients with Anaemia in Tertiary Care Centres. JARHAS, 2(1), pp. 5-8

Hirsch, J.S., Ng, J.H., Ross, D.W., Sharma, P., Shah, H.H., Barnett, R.L., Hazzan, A.D., Fishbane, S., Jhaveri, K.D., Abate, M. and Andrade, H.P., 2020. Acute kidney injury in patients hospitalized with COVID-19. Kidney international, 98(1), pp.209-218.

Huang, C., Wang, Y., Li, X., Ren, L., Zhao, J., Hu, Y., Zhang, L., Fan, G., Xu, J., Gu, X. and Cheng, Z., 2020. Clinical features of patients infected with 2019 novel coronavirus in Wuhan, China. The lancet, 395(10223), pp.497-506.

Husain, Ayan., Anjalee, Chiwhane.,VijendraKirnake., 2020 Non-Invasive Assessment of Liver Fibrosis in Alcoholic Liver Disease, CLINICAL AND EXPERIMENTAL HEPATOLOGY, 6(2),pp. 125-30.

Jain, U., 2020. Effect of COVID-19 on the Organs. Cureus, 12(8).

Latchoumi, T.P., Ezhilarasi, T.P. and Balamurugan, K., 2019. Bio-inspired weighed quantum particle swarm optimization and smooth support vector machine ensembles for identification of abnormalities in medical data. SN Applied Sciences, 1(10), pp.1-10. 
Martínez-Sánchez, J.M., Sureda, X., Fu, M., PérezOrtuño, R., Ballbè, M., López, M.J., Saltó, E., Pascual, J.A. and Fernández, E., 2014. Secondhand smoke exposure at home: assessment by biomarkers and airborne markers. Environmental research, 133, pp.111-116.

Panigada, M., Bottino, N., Tagliabue, P., Grasselli, G., Novembrino, C., Chantarangkul, V., Pesenti, A., Peyvandi, F. and Tripodi, A., 2020. Hypercoagulability of COVID-19 patients in intensive care unit: a report of thromboelastography findings and other parameters of hemostasis. Journal of Thrombosis and Haemostasis, 18(7), pp.1738-1742.

Prakashrao, P.J. and Parwe, S., 2020. A Comparative Clinical Study On The Effect of Anupana Bheda Trivrutta Churna Nitya Virechana In Yakruta Vikara
(Liver Disorders) With Abnormal Liver Function Test. International Journal of Modern Agriculture, 9(3), pp.90-95.

Rume, T. and Islam, S.D.U., 2020. Environmental effects of COVID-19 pandemic and potential strategies of sustainability. Heliyon, p.e04965.

Varga, Z., Flammer, A.J., Steiger, P., Haberecker, M., Andermatt, R., Zinkernagel, A.S., Mehra, M.R., Schuepbach, R.A., Ruschitzka, F. and Moch, H., 2020. Endothelial cell infection and endotheliitis in COVID-19. The Lancet, 395(10234), pp.1417-1418.

WHO.WORLD HEALTH ORGANIZATION; GENEVA: 2020. Coronavirus Disease (COVID-19) pandemic. https://www.who.int/emergencies/diseases/novelcoronavirus-2019. 